\title{
Constraints on Emission Mechanism from the Nulling and Drifting Pulsar PSR B0031-07
}

\author{
B. C. Joshi and M. Vivekanand \\ National Center for Radio Astrophysics, Pune (India)
}

\begin{abstract}
High sensitivity data were obtained on PSR B0031-07 using Ooty Radio Telescope (ORT), which has a single polarization. As this pulsar lies outside the range of other sensitive instruments, such observations are possible only with ORT. Some earlier results, such as the different drift modes, were verified. Our analysis suggests competition between the energies of pairs of drifting sub-pulses and a phase memory across short nulls. The constraints these results put on the theories of pulse emission are enumerated.
\end{abstract}

\section{Results}

The observed distribution of drift rate for PSR B0031-07, computed from 3338 drift bands, has two peaks at $-4.031 \pm 0.011 \mathrm{~ms}$ per period (Class $A$ ) and $-7.771 \pm 0.005$ (Class $B$ ). The two peaks are not strictly harmonic. The average spacing, $P_{2}$, between the drifting sub-pulses appears to increase monotonically with the modulus of drift rate, contrary to popular belief.

The pair of drifting sub-pulses appear to be well separated from each other and their energies are anti-correlated. Our data suggests competition between sub-pulse energies. A significant correlation exists between the sub-pulse phases before (turn-off) and after (turn-on) a null, up-to null durations $\approx 4$ periods, as shown in Figure 1. Assuming that these phases are independent random variables, the expected distributions of total drift (i.e., the difference of turnoff and turn-on phase) for the two modes are inconsistent with the respective observed distributions; this implies a memory of sub-pulse phase across short nulls. Lack of significant correlation for null durations greater than 4 periods suggests that this is a possible time-scale for the phase memory.

Class A drift mode is followed by mostly short duration nulls whereas both short as well as long duration nulls occur after Class B drift bands. Drifting continues during the short nulls, but the nature of drift appears to be different for nulls succeeding the two drift modes. These results suggest different spark mechanisms for these modes.

\section{Discussion}

Sub-pulse drift rates have been observed to be independent of frequency (Nowakowski et al. 1981) suggesting that these are intimately related to the polar cap physics. Furthermore, sub-pulse drift can be understood in terms of 


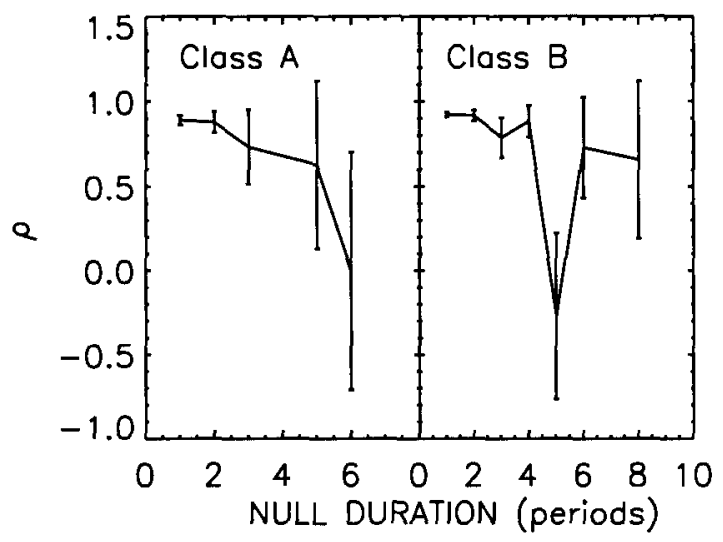

Figure 1. Plot of normalized correlation coefficient for Class A and $B$ modes

the sparking mechanism proposed by Ruderman and Sutherland (1975). However, this model predicts drift speeds that exceed the observed values (Oster et al. 1977). This deficiency can possibly be resolved by (1) invoking new sparking mechanisms, (2) by considering mechanisms such as $\nabla B$ drifts or both. The essential requirement for any such theoretical refinement from our results are

1. It should reproduce the distribution of drift rates for this pulsar as well as other pulsars.

2. It should reproduce the sub-pulse phase memory of the kind seen in PSR B0031-07 and PSR B0809+74. Additionally, if the mechanism involves localized heating of polar cap as proposed by Cheng and Ruderman (1980), it should explain different time-scales for cooling of this spot for PSR B0809+74 ( $10 \sim 20$ periods; Lyne \& Ashworth 1983), PSR B0031-07 ( $4 \sim 5$ periods) and PSR 1944+17 (less than a period; Deich et al. 1986).

3. It should predict different growth rates for adjacent sparks.

4. It should incorporate an increase in apparent spark spacing as a function of drift rate.

\section{References}

Cheng, A. F., \& Ruderman, M. A. 1980, ApJ, 235, 576

Deich, W. T. S. et al. 1986, ApJ, 300, 540

Lyne, A. G., \& Ashworth, M. 1983, MNRAS, 204, 519

Nowakowski, L. et al. 1981, A\&A, 116, 158

Oster, L., Hilton, D. A., \& Sieber, W. 1977, A\&A, 57, 1

Ruderman, M. A., \& Sutherland, P. G. 1975, ApJ, 196, 51 